\title{
The Effect of Hospital Isolation Precautions on Patient Outcomes and Cost of Care: A Multi-Site, Retrospective, Propensity Score-Matched Cohort Study
}

\author{
Kim Tran, BSc' ${ }^{7}$ Chaim Bell, MD PhD ${ }^{2,3}$, Nathan Stall, $M D^{4}$, George Tomlinson, PhD ${ }^{5,6}$, \\ Allison McGeer, MD ${ }^{7,8}$, Andrew Morris, MD SM(Epi) 9,10 , Michael Gardam, MSC MD ${ }^{10,11}$, and \\ Howard B. Abrams, $M D^{1,9,12,13}$
}

\begin{abstract}
'OpenLab, University Health Network, Toronto, ON, Canada; ${ }^{2}$ Institute of Health Policy, Management and Evaluation, University of Toronto, Toronto, ON, Canada; ${ }^{3}$ Department of Medicine, Division of General Internal Medicine, Mount Sinai Hospital, Toronto, ON, Canada; ${ }^{4}$ Core Internal Medicine, University of Toronto, Toronto, ON, Canada; ${ }^{5}$ Dalla Lana School of Public Health, University of Toronto, Toronto, ON, Canada; ${ }^{6}$ Department of Medicine, University Health Network, Toronto, ON, Canada; ${ }^{7}$ Departments of Laboratory Medicine and Pathobiology, Medicine and Dalla Lana School of Public Health, University of Toronto, Toronto, ON, Canada; ${ }^{8}$ Department of Microbiology, Division of Infection Control, Mount Sinai Hospital, Toronto, ON, Canada; ${ }^{9}$ Department of Medicine, University of Toronto, Toronto, ON, Canada; ${ }^{10}$ Department of Medicine, Division of Infectious Diseases, University Health Network and Mount Sinai Hospital, Toronto, ON, Canada; ${ }^{11}$ Infectious Diseases and Medicine, University of Toronto, Toronto, ON, Canada; ${ }^{2}$ Department of Medicine, Division of General Internal Medicine, University Health Network and Mount Sinai Hospital, Toronto, ON, Canada; ${ }^{13}$, Toronto, ON, Canada.
\end{abstract}

BACKGROUND: Isolation precautions have negative effects on patient safety, psychological well-being, and healthcare worker contact. However, it is not known whether isolation precautions affect certain hospitalrelated outcomes.

OBJECTIVE: To examine the effect of isolation precautions on hospital-related outcomes and cost of care.

DESIGN: Retrospective, propensity-score matched cohort study of inpatients admitted to general internal medicine (GIM) services at three academic hospitals in Toronto, Ontario, Canada between January 2010 and December 2012.

PARTICIPANTS: Adult ( $\geq 18$ years of age) patients on isolation precautions for respiratory illnesses and methicillin-resistant Staphylococcus aureus (MRSA) were matched to controls based on propensity scores derived from nine covariates: age, sex, Resource Intensity Weight, number of hospital readmissions within 90 days, total length of stay for hospital admissions within 90 days, site of admission, month of isolation, year of isolation, and Case Mix Group.

MAIN MEASURES: Thirty-day readmission rates and emergency department visits, hospital length of stay, expected length of stay, adverse events, in-hospital mortality, patient complaints, and cost of care in Canadian doll ars (CAD).

KEY RESULTS: A total of 17,649 non-isolated patients were admitted to the participating hospitals during the study period. We identified 1506 patients isolated for respiratory illnesses and 745 patients isolated for MRSA. Compared to non-isolated individuals, those on isolation precautions for respiratory illnesses stayed $17 \%$ longer (95\% CI: $9 \%$, $25 \%$ ), stayed $9 \%$ longer than expected (95\% CI: $3 \%$, $15 \%$ ), and had $23 \%$ higher cost of care (95\% CI: $14 \%$, $32 \%)$. Patients isolated for MRSA had similar outcomes, but

Received August 28, 2015

Revised June 23, 2016

Accepted August 26, 2016

Published online October 17, 2016 they also had a $4.4 \%$ higher (95\% CI: $1.4 \%, 7.3 \%)$ rate of readmission to hospital within 30 days.

CONCLUSIONS: Isolation precautions are associated with adverse effects which may result in poorer hospital outcomes. Balancing the benefits for the many with the harms to the few will be a future challenge.

KEY WORDS: Isolation; Hospital Outcomes.

J Gen Intern Med 32(3):262-8

DOI: $10.1007 / \mathrm{s} 11606-016-3862-4$

c) Society of General Internal Medicine 2016

\section{INTRODUCTION}

Isolation precautions are used for patients hospitalized with known or suspected colonization or infection with pathogens transmitted in hospitals. These precautions may require a private room or placement behind drawn curtains in a multibed room, requiring staff and visitors to wear personal protective equipment, and restricting the movement of patients. ${ }^{1}$ The use of these precautions has been shown to control outbreaks (e.g. severe acute respiratory syndrome $[\mathrm{SARS}]$ ) and, in some circumstances, the transmission of other pathogens. ${ }^{2,3}$ However, such precautions may also be associated with unintended adverse consequences. ${ }^{4,5}$

Isolation precautions negatively affect several dimensions of patient care. Isolated patients have half as much contact with clinicians as do control patients, and are half as likely to be examined by attending physicians on rounds. ${ }^{5-7}$ They are also twice as likely to experience adverse events during hospitalization, and eight times as likely to experience supportive care failure such as falls and pressure ulcers. ${ }^{8,9}$ Studies have reported negative psychological effects of isolation including feelings of loneliness, stigmatization, and increased depression and anxiety. ${ }^{8,10-18}$ Finally, isolated patients experience lower rates of satisfaction with care. ${ }^{8,19}$ 
Some criticisms of these conclusions have suggested that studies to date have not adequately adjusted for the severity of patients' underlying illness, and no studies have examined the trade-off between the known benefits of isolation precautions and their potential risks.

We hypothesize that even with more complete adjustment for patient characteristics and severity of underlying illness, there may be other, unrecognized effects on operational and patient outcomes compared to well-matched controls. We sought to examine whether isolation precautions increased 30-day readmissions and emergency department (ED) visits, hospital length of stay (LOS), expected length of stay (ELOS), adverse events, in-hospital mortality, patient complaints, and cost of care compared to propensity-matched controls.

\section{METHODS}

We conducted a multi-site, retrospective, propensity scorematched cohort study of patients consecutively admitted to general internal medicine (GIM) services using hospital administrative databases in Toronto, Canada. The study was approved by the institutional research ethics boards of the participating hospitals.

\section{Setting}

Patients admitted through the emergency department to GIM at three academic hospitals in Toronto, Canada, between January 2009 and December 2012 were included in the study. All three hospitals are in downtown Toronto, and are tertiary academic institutions affiliated with the University of Toronto. During the 2011 fiscal year, Toronto General (TG), Toronto Western (TW), and Mount Sinai (MS) hospitals admitted 3163 patients, 3250 patients, and 2881 patients, respectively, to their GIM services.

\section{Infection Prevention and Control Practices}

GIM patients are screened at admission for methicillinresistant Staphylococcus aureus (MRSA) colonization using nasal, rectal/groin, and wound swabs. At sites TG and TW, respiratory (droplet and contact) precautions are used for patients admitted with fever and respiratory symptoms; at MS, patients with respiratory or cardiac symptoms are tested for respiratory viruses on admission, and droplet and contact precautions used only for patients with influenza.

An infection control practitioner determines the requirement for isolation precautions. The decision to discontinue isolation is at the discretion of the infection control practitioner, based on improvement in symptoms, confirmatory tests or alternative diagnosis, and/or discussion with attending physician. Information on a patient's isolation status is updated in the patient record. Cases of infectious diseases are confirmed by infection control practitioners and are cross-referenced with the infection control database.

\section{Design and Participants}

Matched cohorts were selected from among all adults ( $\geq 18$ years of age) hospitalized on GIM between January 2009 and December 2012 and who had at least a 2-day LOS. The LOS requirement ensured that patients had adequate exposure to isolation precautions to potentially affect outcomes of care. Two groups of isolated patients were identified: (1) those placed on droplet precautions for respiratory illnesses and (2) those placed on contact precautions for MRSA. We report on these two groups separately, as patients with MRSA are placed on isolation for a longer period of time than patients with respiratory illnesses; as such, the effect of isolation may be more pronounced in the MRSA cohort. Patients in the isolated cohorts include those who had or were suspected of having an infectious disease prior to hospital admission as well as those with hospital-acquired infections; infections acquired during hospitalization represent only a small proportion of cases. The MRSA cohort included patients who were colonized or infected. Patients with no isolation precautions were eligible as controls.

\section{Data Sources}

Variables including patient demographics, clinical characteristics, and outcomes of interest were obtained from two linked hospital administrative databases: the Discharge Abstract Database (DAD) and Enterprise Data Warehouse (EDW). The DAD is an administrative database containing demographic, administrative, and clinical data for hospital discharges that are provided by participating hospitals to the Canadian Institute of Health Information (CIHI). ${ }^{20}$ The EDW is the data repository for clinical and corporate information at TG and TW hospitals. The costs associated with isolated patients and matched controls, in Canadian dollars (CAD), were obtained through the Ministry of Health and Long-Term Care's Ontario Case Costing Initiative (OCCI). OCCI provides participating hospitals with a standardized methodology for the collection of case costing data, allowing for comparisons between hospitals. ${ }^{21-24}$ Only direct costs were considered. Physician costs are not paid from hospital budgets and not included in OCCI case costing.

\section{Study Outcomes}

We collected data on 30-day readmission and ED visits, hospital LOS, ELOS, adverse events, in-hospital mortality, patient complaints, and cost of care. Thirty-day readmission and ED visits were defined as a hospital readmission or ED visit to the same hospital within 30 days of discharge. Hospital LOS was defined as the number of days spent in the hospital from admission until the patient was discharged, transferred to another facility, or died. ELOS is a variable created by CIHI that predicts expected length of hospitalization, adjusting for the reason for hospitalization, comorbidities, age, complications, and inpatient Resource Intensity Weights (RIW); the ELOS values are derived from patient LOS data of all Canadian acute care hospitals within the current 2 years. ${ }^{25} \mathrm{We}$ 
captured the following adverse events through hospital incident reports: falls, infection control incidents, medicationrelated incidents, equipment-related incidents, treatmentrelated incidents, laboratory-related incidents, and operative or invasive incidents. In-hospital mortality was defined as any death occurring during a patient's hospital admission. Patient complaints include any formal complaint made to the hospital patient relations office.

\section{Propensity Score Development}

Propensity score modeling was used to match isolated and non-isolated patients. ${ }^{26-28}$ The propensity score for the likelihood of being placed on isolation precautions was developed using a multivariable logistic regression model. A total of nine covariates were included in the model: age, sex, RIW, number of hospital readmissions within 90 days, total LOS for hospital admissions within 90 days, site of admission, month of isolation, year of isolation, and Case Mix Group (CMG). Two-way interactions were included between the first six covariates, and CMG had a factor for each of the 52 most common groups, which accounted for $75 \%$ of observations. These covariates were all selected as important predictors of isolation for infection control. Controls for each isolated case were sampled with replacement by nearest-neighbor matching using a caliper width of 0.1 standard deviations of the logit of the propensity score. ${ }^{29-31}$ With this approach, isolated patients are randomly ordered, and the first isolated patient is selected and matched to the control patient with the closest propensity score that is within the caliper. If multiple controls are almost equally close to the case, they are all selected and re-weighted to reflect the number of matches. This approach estimates what is called the average treatment effect in the treated (ATT); here, ATT is the effect of isolation on patients who are characteristic of those actually isolated. ${ }^{32}$ Two other effects that could have been estimated, the average treatment effect and the average treatment effect in controls, are of little interest, as they would estimate the effect of isolation in patient groups where isolation is much less likely. Covariate balance between cohorts was assessed graphically and by the standardized difference; meaningful imbalance is indicated by an absolute standardized difference of $0.10 .{ }^{33}$

\section{Statistical Analysis}

Propensity score development, matching, and the analyses below were repeated for the two separate isolated patient groups. We calculated means and percentages for the covariates in the matched and unmatched cohorts. Summaries and comparisons of outcomes were one of three types. Binary outcomes (mortality, readmissions, ED visits, and adverse events) were summarized as percentages and compared with the risk difference. Our continuous outcomes (those related to cost and LOS) were all right-skewed and were summarized by geometric means and compared by the ratio of means. Complaints and adverse events were summarized as rates (number per 100 patient-days) and compared with the difference in rates. All estimates of effects were accompanied by $95 \%$ confidence intervals and $p$ values. Model diagnostics were performed to ensure that all model assumptions were valid. Two-tailed $p$ values of 0.05 or less were considered statistically significant. All analyses were conducted using R software (Vienna, Austria), and matching and estimation of the effects of isolation used the Match package. ${ }^{34}$

\section{Sensitivity Analysis}

To validate results, we also took three other approaches to matching and comparing isolated patients with controls. The first two, stratification and 1:1 nearest-neighbor matching with no ties, used the same main propensity score as in the main analyses. First, stratification involved grouping all isolated patients and all controls into ten strata based on the same propensity score used in our main analysis. Second, we used nearest-neighbor matching without replacement and used only the nearest control to each case to obtain 1:1 matches. The third approach used the package 'twang' in R to estimate the propensity score with generalized boosted regression trees (Table 1). ${ }^{35}$ To obtain the ATT, isolated patients were given a weight of 1 , and all control patients were given a weight of $p s /(1-p s)$, where $p s$ is the patient's propensity score. The effects of isolation were estimated in weighted regression models. We examined the consistency of results across methods, but present full results only for the nearest-neighbor matching within a 0.1 caliper width. Differences between the approaches were also noted.

\section{RESULTS}

A total of 17,649 non-isolated patients were admitted to the participating hospital sites during the study period. We identified 1506 patients isolated for respiratory illnesses and 745 patients isolated for MRSA. Of the MRSA cohort, 20-25\% of cases represented clinical infection, whereas the remainder represented colonization (verbal report, authors MG and AM). The mean age of patients was 67 years, and $50 \%$ were women. The propensitymatched cohorts had similar baseline characteristics (Table 1).

Among the respiratory isolation cohort, 207 (13.7\%) were readmitted to the hospital, and $164(10.9 \%)$ visited the ED within 30 days after discharge. Their mean cost for hospitalization was CAD $\$ 7254$, and their mean length of stay was 8.5 days. Most patients did not experience an adverse event or file a formal complaint with the hospital. Nearly all (99.7\%, $n=1502$ ) patients isolated for respiratory illness were successfully matched to a non-isolated control (Table 2).

Among the cohort with MRSA, 141 (18.9\%) were readmitted to the hospital, and 85 (11.4\%) visited the ED within 30 days of discharge. Their mean cost for hospitalization was CAD $\$ 12,129$, with a mean length of stay of 12.8 days. Overall, $14.4 \%$ of patients experienced an adverse event, and 1.1 patients per 100 admissions filed a formal complaint with the hospital. A total of 737 cases $(98.9 \%)$ had a matched control. 
Table 1 Characteristics of Hospitalized Individuals by Group (pre-match and post-match)

\begin{tabular}{|c|c|c|c|c|c|c|c|c|c|c|c|}
\hline \multirow[t]{3}{*}{ Variable } & \multicolumn{5}{|c|}{ Before matching } & \multicolumn{6}{|c|}{ After matching } \\
\hline & \multirow[t]{2}{*}{$\begin{array}{l}\text { Control } \\
(n=17,649)\end{array}$} & \multicolumn{2}{|c|}{$\begin{array}{l}\text { MRSA } \\
(n=745)\end{array}$} & \multicolumn{2}{|c|}{$\begin{array}{l}\text { Respiratory } \\
\text { illness }(n= \\
1506)\end{array}$} & \multicolumn{3}{|c|}{$\begin{array}{l}\text { MRSA } \\
(n=737) \\
8 \text { cases dropped }\end{array}$} & \multicolumn{3}{|c|}{$\begin{array}{l}\text { Respiratory illness } \\
(n=1502) \\
4 \text { cases dropped }\end{array}$} \\
\hline & & Value & $\begin{array}{l}\text { Std. } \\
\text { diff. }\end{array}$ & Value & $\begin{array}{l}\text { Std. } \\
\text { diff. }\end{array}$ & Isolated & Control & $\begin{array}{l}\text { Std. } \\
\text { diff. }\end{array}$ & Isolated & Control & $\begin{array}{l}\text { Std. } \\
\text { diff. }\end{array}$ \\
\hline Age (years) & 67.9 & 69.0 & 0.08 & 71.7 & 0.29 & 69.0 & 69.0 & 0.00 & 71.7 & 70.6 & 0.06 \\
\hline Male $(\%)$ & 50 & 58 & 0.22 & 53 & 0.10 & 57 & 58 & 0.00 & 53 & 55 & -0.03 \\
\hline $\begin{array}{l}\text { Number of admissions in } \\
\text { previous } 90 \text { days }\end{array}$ & 0.24 & 0.38 & 0.33 & 0.27 & 0.08 & 0.38 & 0.39 & -0.01 & 0.27 & 0.28 & -0.01 \\
\hline $\begin{array}{l}\text { Length of stay in previous } 90 \\
\text { days (days) }\end{array}$ & 2.12 & 4.38 & 0.36 & 2.95 & 0.13 & 3.88 & 3.4 & 0.04 & 2.95 & 2.94 & 0.00 \\
\hline Resource Intensity Weight & 1.52 & 2.87 & 0.76 & 1.84 & 0.21 & 2.3 & 2.08 & 0.07 & 1.8 & 1.84 & -0.01 \\
\hline $\begin{array}{l}\text { CMG196 - heart failure } \\
\text { without cardiac catheter (\%) }\end{array}$ & 6 & 7 & 0.06 & 5 & -0.04 & 7 & 7 & 0.01 & 5 & 5 & 0.00 \\
\hline $\begin{array}{l}\text { CMG138 - viral/unspecified } \\
\text { pneumonia }(\%)\end{array}$ & 3 & 6 & 0.28 & 25 & 1.47 & 7 & 7 & -0.02 & 25 & 24 & 0.04 \\
\hline $\begin{array}{l}\text { CMG139 - chronic obstructive } \\
\text { pulmonary disease }(\%)\end{array}$ & 3 & 6 & 0.29 & 15 & 0.92 & 6 & 7 & -0.02 & 15 & 16 & -0.03 \\
\hline $\begin{array}{l}\text { CMG254 - gastrointestinal } \\
\text { hemorrhage }(\%)\end{array}$ & 3 & 2 & -0.10 & 0 & -0.25 & 2 & 2 & 0.00 & 0 & 0 & N/A \\
\hline $\begin{array}{l}\text { CMG487 - lower urinary tract } \\
\text { infection }(\%)\end{array}$ & 4 & 7 & 0.24 & 3 & -0.02 & 7 & 7 & -0.02 & 3 & 3 & 0.01 \\
\hline
\end{tabular}

MRSA methicillin-resistant Staphylococcus aureus, CMG Case Mix Group

\section{Outcomes for Patients Isolated for Respiratory Illness}

Individuals isolated for respiratory illnesses were not significantly more likely to experience a 30-day readmission or ED visit, death, or adverse event during hospitalization or to file a formal complaint compared to non-isolated individuals (Table 3). However, those on isolation precautions for respiratory illnesses stayed $17 \%$ longer (LOS, 8.5 days vs. 7.6 days; $95 \%$ CI: 1.09, 1.12), stayed $9 \%$ longer than expected, (LOS/ELOS, 1.08 vs. 1.01; $95 \%$ CI: 1.03 , 1.15 ), and had $23 \%$ higher cost of care (direct cost, CAD $\$ 7194$ vs. CAD \$6294; $95 \%$ CI: 1.14, 1.32) than non-isolated individuals. These findings are consistent across all approaches.

\section{Outcomes for Patients Isolated for MRSA}

Patients isolated for MRSA were $4.4 \%$ more likely than nonisolated individuals to be readmitted within 30 days after discharge (rate, $19.0 \%$ vs. $14.7 \%$; $95 \%$ CI: $1.4 \%, 7.3 \%$; Table 4). There were no significant differences in 30-day ED visits, formal complaints, or inpatient mortality rates between the cohorts.

Similar to patients with respiratory illness, patients isolated for MRSA stayed $30 \%$ longer (LOS, 11.9 days vs. 9.1 days; $95 \%$ CI: $1.22,1.39)$, stayed $13 \%$ longer than expected, (LOS/ ELOS, 1.3 vs. $1.2 ; 95 \%$ CI: $1.07,1.20)$, and had $43 \%$ higher cost of care (direct cost, CAD \$11,009 vs. CAD \$7670; $95 \%$

Table 2 Descriptive Statistics for Outcome Variables Comparing Isolated to Non-Isolated Cohorts (pre-match)

\begin{tabular}{|c|c|c|c|c|c|}
\hline \multirow[t]{2}{*}{$\overline{\text { Outcome }}$} & \multirow[t]{2}{*}{ Control } & \multicolumn{2}{|l|}{ MRSA } & \multicolumn{2}{|c|}{ Respiratory illness } \\
\hline & & Isolated & $p$ value & Isolated & $p$ value \\
\hline Inpatient mortality, \% & 5.7 & 8.2 & 0.0048 & 6.9 & 0.0531 \\
\hline 30-day readmission, \% & 12.9 & 18.9 & $<0.0001$ & 13.7 & 0.4297 \\
\hline 30-day ED visit, \% & 12.8 & 11.4 & 0.2978 & 10.9 & 0.0379 \\
\hline Readmission or ED visit, \% & 18.4 & 22.6 & 0.0049 & 17.3 & 0.3260 \\
\hline ELOS (days) & 7.3 & 10.2 & $<0.0001$ & 8.3 & $<0.0001$ \\
\hline $\operatorname{LOS}$ (days) & 7.6 & 12.8 & $<0.0001$ & 8.5 & $<0.0001$ \\
\hline LOS/ELOS & 1.2 & 1.4 & 0.0001 & 1.1 & 0.0086 \\
\hline Cost, CAD & 6146 & 12129 & $<0.0001$ & 7254 & $<0.0001$ \\
\hline Cost per day, CAD & 852 & 946 & $<0.0001$ & 855.7 & 0.0025 \\
\hline \multicolumn{6}{|l|}{ Adverse events, $n / 100$} \\
\hline Fall & 5.6 & 12.1 & $<0.0001$ & 4.2 & 0.0231 \\
\hline Medication incident & 1.5 & 2.1 & 0.1741 & 2.1 & 0.0676 \\
\hline Laboratory incident & 0.4 & 0.3 & 0.5622 & 1.0 & 0.0035 \\
\hline Treatment incident & 0.4 & 0.5 & 0.5731 & 0.6 & 0.2720 \\
\hline Infection control incident & 0.1 & 1.9 & $<0.0001$ & 1.0 & $<0.0001$ \\
\hline Safety incident & 0.3 & 0.3 & 0.8529 & 0.3 & 0.8628 \\
\hline Equipment incident & 0.1 & 0.7 & 0.0010 & 0.5 & 0.0012 \\
\hline Any adverse event & 6.8 & 14.4 & $<0.0001$ & 9.2 & 0.0012 \\
\hline Patient complaints, $n / 100$ & 1.0 & 1.1 & 0.9113 & 2.7 & $<0.0001$ \\
\hline
\end{tabular}

MRSA methicillin-resistant Staphylococcus aureus, ED emergency department, ELOS expected length of stay, LOS length of stay 
Table 3 Respiratory Isolation Cohort - Outcome Variables Comparing Isolated to Non-Isolated Cohorts (post-match)

\begin{tabular}{|c|c|c|c|c|c|}
\hline$\overline{\text { Outcome }}$ & Isolated & Control & Effect & $\begin{array}{l}95 \% \\
\text { CI }\end{array}$ & $p$ value \\
\hline Inpatient mortality*, \% & 6.9 & 8.5 & -1.6 & $-3.9,0.6$ & 0.1578 \\
\hline 30-day readmission*, \% & 13.7 & 15.7 & -2.0 & $-5.0,1.1$ & 0.2088 \\
\hline 30-day ED visit*, \% & 10.9 & 11.2 & -0.2 & $-2.9,2.5$ & 0.8664 \\
\hline Readmission or ED visit* $\%$ & 17.4 & 18.5 & -1.1 & $-4.5,2.2$ & 0.5005 \\
\hline $\operatorname{ELOS}^{\dagger}$ (days) & 8.3 & 7.8 & 1.07 & $1.03,1.12$ & 0.0019 \\
\hline $\operatorname{LOS}^{\dagger}$ (days) & 8.5 & 7.6 & 1.17 & $1.09,1.25$ & $<0.0001$ \\
\hline LOS/ELOS & 1.08 & 1.01 & 1.09 & $1.03,1.15$ & 0.0046 \\
\hline $\operatorname{Cost}^{\dagger}, \mathrm{CAD}$ & 7194 & 6294 & 1.23 & $1.14,1.32$ & $<0.0001$ \\
\hline Cost per day ${ }^{\dagger}$, CAD & 855 & 826 & 1.05 & $1.02,1.08$ & 0.0030 \\
\hline \multicolumn{6}{|l|}{ Adverse events,$n / 100$} \\
\hline Fall & 4.2 & 5.1 & -0.9 & $-3.2,1.3$ & 0.4236 \\
\hline Medication incident & 2.1 & 1.6 & 0.5 & $-0.7,1.7$ & 0.4122 \\
\hline Laboratory incident & 0.9 & 0.6 & 0.3 & $-0.7,1.2$ & 0.5824 \\
\hline Treatment incident & 0.6 & 0.7 & -0.1 & $-0.8,0.6$ & 0.7554 \\
\hline Infection control incident & 1.0 & 0.6 & 0.4 & $-0.4,1.2$ & 0.2831 \\
\hline Safety incident & 0.3 & 0.1 & 0.3 & $-0.6,1.2$ & 0.5418 \\
\hline Equipment incident & 0.4 & 0.2 & 0.2 & $-0.4,0.8$ & 0.4399 \\
\hline Any adverse event & 9.1 & 8.9 & 0.2 & $-2.9,3.4$ & 0.8830 \\
\hline Patient complaints ${ }^{*}, n / 100$ & 2.6 & 1.0 & 1.6 & $-0.2,3.4$ & 0.0839 \\
\hline
\end{tabular}

*Reported as percentages; effect is the difference in percentage between the isolated and non-isolated cohorts

${ }^{\dagger}$ Reported as geometric means; effect is the ratio of means between the isolated and non-isolated cohorts

Reported as rates per 100 admissions; effect is the absolute differences in the rates per 100 patients between isolated and non-isolated cohorts

ED emergency department, ELOS expected length of stay, LOS length of stay

CI: $1.33,1.54)$ compared to matched controls. These findings were consistent across all statistical approaches.

\section{DISCUSSION}

The results of our study show that in comparison to non-isolated patients, patients isolated for respiratory illnesses and MRSA had longer lengths of hospital stay, stayed in the hospital longer than expected, and had higher costs. Differences also existed between the respiratory illness and MRSA cohorts. Patients isolated for MRSA were more likely to be readmitted to the hospital within 30 days after discharge, which was not found for patients isolated for respiratory illnesses. Neither isolated cohort was more likely to experience a 30-day ED visit or death or to file a formal complaint compared to matched controls.

Evidence is lacking as to whether respiratory precautions reduce nosocomial spread of respiratory disease; therefore, it is difficult to assess benefits versus adverse impacts of this practice.

Table 4 MRSA Isolation Cohort - Outcome Variables Comparing Isolated to Non-Isolated Cohorts (post-match)

\begin{tabular}{|c|c|c|c|c|c|}
\hline Outcome & Isolated & Control & Effect & $95 \% \mathrm{CI}$ & p value \\
\hline Inpatient mortality*, \% & 8.0 & 7.0 & 1.0 & $-1.0,3.1$ & 0.3276 \\
\hline 30-day readmission*, \% & 19.0 & 14.7 & 4.4 & $1.4,7.3$ & 0.0041 \\
\hline 30-day ED visit*, \% & 11.4 & 11.7 & -0.3 & $-2.6,2.1$ & 0.8228 \\
\hline Readmission or ED visit*, \% & 22.7 & 19.3 & 3.4 & $0.2,6.6$ & 0.0366 \\
\hline $\operatorname{ELOS}^{\dagger}$ (days) & 9.8 & 8.4 & 1.15 & $1.09,1.20$ & $<0.0001$ \\
\hline $\operatorname{LOS}^{\dagger}$ (days) & 11.9 & 9.1 & 1.30 & $1.22,1.39$ & $<0.0001$ \\
\hline LOS/ELOS & 1.3 & 1.2 & 1.13 & $1.07,1.20$ & $<0.0001$ \\
\hline $\mathrm{Cost}^{\dagger}, \mathrm{CAD}$ & 11009 & 7670 & 1.43 & $1.33,1.54$ & $<0.0001$ \\
\hline Cost per day ${ }^{\dagger}, \mathrm{CAD}$ & 938 & 868 & 1.10 & $1.06,1.13$ & $<0.0001$ \\
\hline \multicolumn{6}{|l|}{ Adverse events $n / 100$} \\
\hline Fall & 10.3 & 8.0 & 2.3 & $-1.5,6.1$ & 0.2309 \\
\hline Medication incident & 2.2 & 2.4 & -0.3 & $-1.7,1.2$ & 0.7274 \\
\hline Laboratory incident & 0.27 & 0.33 & -0.1 & $-0.4,0.3$ & 0.7834 \\
\hline Treatment incident & 0.4 & 0.5 & -0.1 & $-0.6,0.4$ & 0.7620 \\
\hline Infection control incident & 1.8 & 0.11 & 1.7 & $0.7,2.6$ & 0.0007 \\
\hline Safety incident & 0.1 & 0.3 & -0.2 & $-0.5,0.1$ & 0.2006 \\
\hline Equipment incident & 0.5 & 0.2 & 0.3 & $-0.4,1$ & 0.3629 \\
\hline Any adverse event & 12.4 & 10.7 & 1.7 & $-2.3,5.7$ & 0.4100 \\
\hline Patient complaints ${ }^{*}, n / 100$ & 1.09 & 1.25 & -0.2 & $-1.3,1$ & 0.7864 \\
\hline
\end{tabular}

*Reported as percentages; effect is the difference in percentage between the isolated and non-isolated cohorts

${ }^{*}$ Reported as geometric means; effect is the ratio of means between the isolated and non-isolated cohorts

Reported as rates per 100 admissions; effect is the absolute difference in the rates per 100 patients between isolated and non-isolated cohorts

MRSA methicillin-resistant Staphylococcus aureus, ED emergency department, ELOS expected length of stay, LOS length of stay 
For MRSA, the reproductive ratio, R(A), for a single admission of an MRSA patient not on precautions is about 0.67 . The reproductive ratio for MRSA rises above 1 only when readmissions are included, since patients can be colonized for years. Precautions reduce the R(A) by $>90 \% .^{36,37}$ Thus, each patient in precautions from admission, as opposed to nonisolated, would result-from that admission-in about 0.67 $\times 0.35=0.23$, i.e. just under one quarter of one infection. If more than one admission is counted, the number of prevented infections increases. In addition, if started early enough, the intervention prevents not only the first generation of transmission, but also later ones, hence further increasing the benefits. 36,37

However, isolation precautions may negatively impact several dimensions of patient care. Our study adds to knowledge in this area by noting that isolation precautions are associated with certain negative hospital-related outcomes. Previous studies have reported negative psychological effects, more adverse events, and less healthcare provider contact for isolated patients. ${ }^{6-11,13,16-18}$ This may lead to a disproportionate amount of 'hospitalization-induced disability', i.e. the impact of the hospitalization itself, with subsequent de-conditioning, over and above the severity of the illness, resulting in loss of function, prolonging hospital stay, and possibly leading to an increased 30-day readmission rate.

Others have reported higher rates of adverse events, especially supportive care failures such as falls, for isolated versus non-isolated patients. ${ }^{8,10}$ Our study does not fully support these findings, as we found no statistically significant difference in fall rates; it is possible that our propensity score-matched design better adjusted for severity of illness than studies that found a significant difference in these rates. Our work contributes novel information on the effect of isolation for respiratory illnesses and MRSA on various hospital-related outcomes, specifically 30-day readmission rates and ED visits, in-hospital mortality, LOS, ELOS, cost of care, adverse events, and patient complaints.

Our work has limitations. Although we studied three separate hospitals from two different health systems, they are all University of Toronto teaching hospitals located in downtown Toronto, Canada. Hence, this may reduce the generalizability of the results.

When comparing isolated and non-isolated patients, caution must be used in attributing differences in outcomes and cost of care to the conditions of isolation rather than illness severity. We attempted to mitigate this by using a propensity scorematched design. Additionally, the literature suggests that MRSA infections lead to worse outcomes, higher costs, and longer lengths of stay than those observed for methicillinsusceptible infections - thus, some of the negative outcomes here may be due to the underlying infection and/or an inherent but unmeasured difference in patient status, and not the isolation practice itself. ${ }^{38,39}$ Another limitation noted in 'Methods' is that the practice of isolation for infection control differs between sites. At sites 1 and 2, respiratory (droplet and contact) precautions are used for patients admitted with fever and respiratory symptoms; at site 3, patients with respiratory or fever with cardiac symptoms are tested for respiratory viruses on admission, whereas droplet and contact precautions are used only for patients with influenza. Additionally, the sites differ in their requirement for hospital staff to wear masks when caring for patients colonized or infected with MRSA. Finally, we acknowledge that data for 30-day hospital readmissions are limited to the hospital the patient was originally discharged from. However, in our region, two-thirds of readmissions are to the same site as the initial hospitalization. ${ }^{40}$ Moreover, the potential for missing readmission data should be equally distributed across both isolated and non-isolated patients.

Our results have some important implications. Isolation precautions are seen as important public health and infection control measures. Despite this expected benefit, we found that these practices are associated with unintended consequences. It is possible that infection control procedures for hospitalized patients contribute to poorer outcomes that they experience, and hence more attention needs to be paid to mitigating the risks associated with isolation.

The finding of higher costs for isolated patients is important. Many healthcare institutions are remunerated through activitybased funding, which incorporates patient volume and case mix. However, isolated patients are staying longer than expected based on this methodology, and thus institutions may not be sufficiently compensated to care for them if this characteristic is not considered. Institutional remuneration schemes may require re-evaluation so that hospitals are properly compensated for isolated patients, who consist of a significant minority of the patient population on GIM wards.

\section{CONCLUSION}

Isolation precautions for infection control purposes are considered an important public health intervention. However, isolation may adversely affect the isolated individual and, in our study, was associated with longer LOS, higher costs, and increased hospital readmissions at 30 days for patients with MRSA. Balancing the benefits for the many with the harms to the few is a future challenge for hospital isolation practices.

\section{Acknowledgments:}

This study was funded through Health Research Grant no. 13-28 provided by the Physicians' Services Incorporated Foundation. Kim Tran and George Tomlinson had full access to all the data in the study and take responsibility for the integrity of the data and the accuracy of the data analysis.

Authors' Contributions: All authors provided substantial contributions to conception or design of the work, or the acquisition, analysis, or interpretation of data for the work; drafting of the work or revising it critically for important intellectual content; and final approval of the version to be published. All authors agree to be accountable for all aspects of the work in ensuring that questions related to the accuracy 
or integrity of any part of the work are appropriately investigated and resolved.

Corresponding Author: Howard B. Abrams, MD; , TGH EN14-218, 200 Elizabeth Street, Toronto, ON M5G 2C4, Canada (e-mail: Howard.abrams@uhn.ca).

\section{Compliance with Ethical Standards:}

Conflict of Interest: The authors declare that they do not have a conflict of interest.

\section{REFERENCES}

1. Garner JS. Guideline for isolation precautions in hospitals. The Hospital Infection Control Practices Advisory Committee. Infect Control Hosp Epidemiol. 1996;17(1):53-80.

2. Seto WH, Tsang D, Yung RW, et al. Effectiveness of precautions against droplets and contact in prevention of nosocomial transmission of severe acute respiratory syndrome (SARS). Lancet. 2003;361:1519-20.

3. Loveday HP, Pellowe CM, Jones SRLJ, Pratt RJ. A systematic review of the evidence for interventions for the prevention and control of meticillinresistant Staphylococcus aureus (1996-2004): report to the Joint MRSA Working Party (Subgroup A). J Hosp Infect. 2006;63(Suppl 1):S45-70.

4. Burke JP. Infection control - a problem for patient safety. N Engl J Med. 2003;348(7):651-6.

5. Kirkland KB. Taking off the gloves: toward a less dogmatic approach to the use of contact isolation. Clin Infect Dis. 2009;48(6):766-71.

6. Kirkland KB, Weinstein JM. Adverse effects of contact isolation. Lancet. 1999;354(9185): 1177-8.

7. Saint S, Higgins LA, Nallamothu BK, Chenoweth C. Do physicians examine patients in contact isolation less frequently? A brief report. Am J Infect Control. 2003;31(6):354-6.

8. Stelfox HT, Bates DW, Redelmeier DA. Safety of patients isolated for infection control. JAMA. 2003;290(14):1899-905.

9. Spence MR, McQuaid M. The interrelationship of isolation precautions and adverse events in an acute care facility. Am J Infect Control. 2011;39(2):154-5.

10. Catalano G, Houston SH, Catalano MC, et al. Anxiety and depression in hospitalized patients in resistant organism isolation. South Med J. 2003;96(2):141-5.

11. Gammon J. Analysis of the stressful effects of hospitalisation and source isolation on coping and psychological constructs. Int $J$ Nurs Pract. 1998;4(2):84-96.

12. Maunder R, Hunter $\mathbf{J}$, Vincent $\mathbf{L}$, et al. The immediate psychological and occupational impact of the 2003 SARS outbreak in a teaching hospital. CMAJ. 2003; 168(10):1245-51.

13. Tarzi S, Kennedy P, Stone S, Evans M. Methicillin-resistant Staphylococcus aureus: psychological impact of hospitalization and isolation in an older adult population. J Hosp Infect. 2001;49(4):250-4.

14. Day HR, Perencevich EN, Harris AD, Himelhoch SS, Brown CH, GruberBaldini AL, et al. Do contact precautions cause depression? A two-year study at a tertiary care medical centre. J Hosp Infect. 2011;79(2):103-7.

15. Oldman T. Isolated case. Nurs Times. 1998;94:67-70.

16. Rees J. Psychological effects of isolation nursing (1): mood disturbance. Nurs Stand. 2000; 14:35e38.

17. Kennedy P, Hamilton LR. Psychological impact of the management of methicillin-resistant Staphylococcus aureus (MRSA) in patients with spinal cord injury. Spinal Cord. 1997;35:617-9.

18. Wilkins EG, Ellis ME, Dunbar EM, Gibbs A. Does isolation of patients with infections induce mental illness? J Infect. 1988;17:43e47.

19. Gasink LB, Singer K, Fishman NO, et al. Contact isolation for infection control in hospitalized patients: is patient satisfaction affected? Infect Control Hosp Epidemiol. 2008;29(3):275-8.

20. Canadian Institute for Health Information. Discharge Abstract Database (DAD) metadata. http://www.cihi.ca/CIHI-ext-portal/internet/en/ document/types+of+care/hospital+care/acute+care/dad_metadata Published 2014. Accessed January 2015.

21. Ontario Ministry of Health and Long-Term Care. Ontario Case Costing Initiative. https://hsimi.on.ca/hdbportal/ocdm. Published 2011. Accessed August 16, 2012.

22. Cohen E, Lacombe-Duncan A, Spalding $\mathbf{K}$, et al. Integrated complex care coordination for children with medical complexity: a mixed-methods evaluation of tertiary care-community collaboration. BMC Health Serv Res. 2012;12:366.

23. Hwang SW, Weaver J, Aubry T, Hoch JS. Hospital costs and length of stay among homeless patients admitted to medical, surgical, and psychiatric services. Med Care. 2011;49(4):350-354.

24. O'Brien BJ, Willan A, Blackhouse G, Goeree R, Cohen M, Goodman S. Will the use of low-molecular-weight heparin (enoxaparin) in patients with acute coronary syndrome save costs in Canada? Am Heart J. 2000; 139(3):423-429.

25. Perry S, Homan C. Use of case mix tools for utilization management and planning. Sud Health Technol Inf. 2009;143:496-500.

26. Rubin DB. The design versus the analysis of observational studies for causal effects: parallels with the design of randomized trials. Stat Med. 2007;26:20-36.

27. Austin PC. Propensity-score matching in the cardiovascular surgery literature from 2004 to 2006: a systematic review and suggestions for improvement. J Thorac Cardiovasc Surg. 2007;134:1128-1135.

28. Winkelmayer wC, Kurth T. Propensity scores: help or hype? Nephrol Dial Transplant. 2004; 19:1671-1673.

29. Austin PC. Some methods of propensity-score matching had superior performance to others: results of an empirical investigation and Monte Carlo simulations. Biom J. 2009;51(1):171-84.

30. Austin PC. An introduction to propensity score methods for reducing the effects of confounding on observational studies. Multivariate Behav Res. 2011;46:399-424.

31. D'Agostino R. Tutorial in biostatistics: propensity score methods for bias reduction in the comparison of a treatment to a non-randomized control group. Stat Med. 1998;17(19):2265-81.

32. Rosenbaum PR, Rubin DB. Constructing a control group using multivariate matched sampling methods that incorporate the propensity score. Am Stat. 1985;39(1):33-8.

33. Austin PC. Balance diagnostics for comparing the distribution of baseline covariates between treatment groups in propensity-score matched samples. Stat Med. 2009;28:3083-3107.

34. Sekhon JS. Multivariate and propensity score matching software with automated balance optimization: the matching package for R. J Stat Softw. 2011;42(7): 1-52.

35. Ridgeway G, McCaffrey D, Morral A, Ann B, Burgette L. Toolkit for weighting and analysis of non-equivalent groups. https://cran.r-project. org/web/packages/twang/vignettes/twang.pdf Published April 282016. Accessed June 26, 2016

36. Jernigan JA, Titus MG, Groschel DH, Getchell-White S, Farr BM. Effectiveness of contact isolation during a hospital outbreak of methicillinresistant Staphylococcus aureus. Am J Epidemiol. 1996; 143(5):496-504.

37. Geffers C, Farr BM. Risk of transmission of nosocomial methicillinresistant Staphylococcus aureus (MRSA) from patients colonized with MRSA. Infect Control Hosp Epidemiol. 2005;26(2):114-5.

38. Filice GA, Nyman JA, Lexau C, Lees CH, Bockstedt LA, Como-Sabetti $\mathbf{K}$, Lesher LJ, Lynfield R. Excess costs and utilization associated with methicillin resistance for patients with Staphylococcus aureus infection. Infect Control Hosp Epidemiol. 2010;31(4):365-73.

39. Cosgrove SE, Qi Y, Kaye KS, Harbarth S, Karchmer AW, Carmeli Y. The impact of methicillin resistance in Staphylococcus aureus bacteremia on patient outcomes: mortality, length of stay, and hospital charges. Infect Control Hosp Epidemiol. 2005;26(2):166-74.

40. Gruneir A, Dhalla IA, Van Walraven C, et al. Unplanned readmissions after hospital discharge among patients identified as being at high risk for readmission using a validated predictive algorithm. Open Med. 2011;5(2):e104-e111. 